\title{
Operator product expansion and confinement
}

\author{
V. Shevchenko \\ Institute for Theoretical Physics, Utrecht University, Leuvenlaan 4, 3584 CE Utrecht, The Netherlands \\ and Institute of Theoretical and Experimental Physics, B. Cheremushkinskaya 25, 117218 Moscow, Russia \\ Yu. Simonov \\ Institute of Theoretical and Experimental Physics, B. Cheremushkinskaya 25, 117218 Moscow, Russia
}

(Received 4 October 2001; published 1 April 2002)

\begin{abstract}
The operator product expansion (OPE) technique is analyzed in Abelian and non-Abelian field theoretical models with confinement. Special attention is paid to the regimes where the nonzero virtuality of vacuum fields is felt by external currents. It is stressed that despite the fact that the physics of confinement is sometimes considered as being caused by "soft" fields, it can exhibit pronounced "hard" effects in OPE.
\end{abstract}

DOI: $10.1103 /$ PhysRevD.65.074029

PACS number(s): 12.38.Aw, 11.15.- q

\section{INTRODUCTION}

The inclusion of nonperturbative contributions [1] (proportional to the gauge-invariant local condensates) in standard perturbative operator product expansion (OPE) allows one to [2] formulate a powerful method of QCD sum rules [1] (for reviews, see [3-5]). Nevertheless, some questions about the method were formulated in the original papers $[6,7]$ and still remain unanswered.

In particular, a relation between the property of confinement and the structure of the sum-rule series has never been clearly established. On the one hand, one could guess that confinement appears as a result of the partial summation of some OPE subseries, while on the other hand, confinement itself might introduce some new unconventional terms in OPE series, with the structure different from the standard form.

The phenomenological implication of such new terms, e.g., $O\left(1 / Q^{2}\right)$, was investigated in [8], where it was related to the short-distance nonperturbative physics. The authors of [9-12] checked the role of confinement for QCD sum rules exploiting nonrelativistic solvable models, and exact results for Green's functions were compared to the sum-rule results.

Especially popular is the example of nonrelativistic particles in the oscillator potential, with the Euclidean shorttime expansion of the Green's function [for a detailed discussion of the two-dimensional (2D) case, see [5]; for the 3D case, see [9]],

$$
G^{\mathrm{osc}}(T)=\frac{m}{2 \pi T}\left(1-\frac{(\omega T)^{2}}{6}+\frac{7}{360}(\omega T)^{4}+\cdots\right)
$$

Here the first term comes from the free Green's function while the next terms play the role of "condensates," i.e., they identify Borel mass $\varepsilon=1 / T$, One has the typical OPE structures $\omega^{2} / \varepsilon^{2}$ and $\omega^{4} / \varepsilon^{4}$.

The result (1) has widely been used as an argument that confinement (i.e., long-distance soft physics) cannot modify the standard OPE, and confinement effects should be looked for in the partial sums of the type $\sum_{n=0}^{\infty} c_{n}\left(Q^{2}\right)\left\langle D^{n} F(0) D^{n} F(0)\right\rangle$.
In what follows, we shall demonstrate explicitly that confinement modifies the standard OPE for a relativistic quark Green's function; new terms appear, which bring unusual power terms in OPE.

It will be shown that the expansion (1) is typical for nonrelativistic potential Green's functions, while for relativistic particles in the confining fields (or in the confining potential) a specific long-distance instability (divergence) occurs in the perturbative expansion, which could lead to new power terms.

Let us stress from the beginning an important difference between OPE in coordinate and momentum spaces, which was discussed already in $[1,13]$ and which will be seen clearly in what follows. Studying the small- $x$ expansion of a product of two operators $\langle T\{J(0) J(x)\}\rangle$ when $x \rightarrow 0$, one observes that in the relativistic case (contrary to the nonrelativistic one), a small value of $x$ does not confine the virtualities of the internal lines in the corresponding diagrams in any way. Virtual particles created and annihilated by operators $J$ can travel over large distances in coordinate space for any small $x$. As a result, in confining theory the product of operators taken at two neighboring points carries information about large-distance behavior of a theory even if $x$ is much smaller than the typical confinement scale $\lambda^{-1}$.

To clarify the mechanism of this phenomenon, we start in the next section with the Green's function of a relativistic quark in the linear confining potential of a static antiquark, corresponding to the Dirac equation with a scalar linear potential. We shall expand Green's function in powers of string tension (or equivalently in powers of Euclidean time $T$ ) and find explicitly a new dominant term at small $T$ and estimate other terms. Comparison with the corresponding nonrelativistic Green's function is done and demonstrates that no unusual terms appear in the latter case, the expansion being essentially of the same type as in Eq. (1). The reason for that is traced to the structure of the nonrelativistic free Green's function, for which spatial deflection of particle $\Delta x$ is limited by the time elapsed $\Delta t, \Delta x \sim \sqrt{\Delta t / m}$.

The situation is different, however, in momentum space. Large external momentum $Q$ plays the role of infrared cutoff, and if it is much greater than particle mass $m$ and nonperturbative scale $\lambda$, one can perform systematic expansions over 
$m^{2} / Q^{2}$ and $\lambda^{2} / Q^{2}$. This is how the standard OPE technique works. Nevertheless, the remaining problem in this case is to determine the structure of the latter, nonperturbative subseries. The problem here is that in real QCD, there are several different nonperturbative scales. The best known scales are given by nonperturbative quark and gluon [1] condensates $\langle\bar{\psi} \psi\rangle,\left\langle F_{\mu \nu} F_{\mu \nu}\right\rangle$. One can include in analysis higher irreducible condensates as well. Another important scale is given by condensate virtualities [see expressions (21) and (22) below]. So even remaining in the standard OPE framework, one can attempt to incorporate the different subseries in the full $\lambda^{2} / Q^{2}$ expansion. It will be shown below how this problem is solved in particular cases.

Moreover, we present a few examples in Sec. VI where OPE in momentum space starts from the terms that nontrivially account for (monopole) condensate virtuality and hence would be considered as subleading in the conventional expansion.

The field-theoretical models are discussed in Sec. III, where the QCD equations for the heavy-light system obtained in the limit of large $N_{c}$ in [14] are discussed.

It is shown, in particular, based on the subsequent results in $[15,16]$, that exact equations have a nonlinear kernel, which at large spatial distances reduces to the linear confining term $\sigma|\vec{r}|$, and hence the expansion of the Green's function reduces to the potential example considered in Sec. II.

We briefly consider Abelian models in Sec. VI, such as QED with monopoles, and study the influence of confinement on the short-time behavior of Green's functions. We also discuss various approaches related to OPE, such as the Feynman-Schwinger proper time method (Sec. V), and spectral representations of Green's functions (Sec. VII), and we study the interplay between confinement and OPE in these frameworks. Finally, we present a short conclusion and outlook.

\section{RELATIVISTIC GREEN'S FUNCTION OF A CONFINED QUARK}

We study Green's function of the Dirac equation in the Euclidean space-time,

$$
-i(\hat{\partial}+m+\sigma|\vec{x}|) S(x, y)=\delta^{(4)}(x-y) .
$$

In what follows, we shall study the function $S\left(\vec{x}=0 ; x_{4}\right.$ $\left.=0 ; \vec{y}=0, y_{4}=T\right) \equiv S(T)$ as a function of $T$ at small values of $T$.

The free Green's function $S_{0}(x-y)$ can be written as

$$
\begin{aligned}
S_{0}(x) & =\int \frac{d^{4} p}{(2 \pi)^{4}} \frac{\exp (i p x)}{p^{2}+m^{2}}(\gamma p+i m) \\
& =i(m-\hat{\partial})\left\langle 0\left|\left(m^{2}-\partial^{2}\right)^{-1}\right| x\right\rangle \\
& =i(m-\hat{\partial}) \frac{m}{4 \pi^{2}} \frac{K_{1}(m x)}{x} \\
& =i\left(m-\frac{\hat{x}}{x} \frac{\partial}{\partial x}\right) \frac{m}{4 \pi^{2}} \frac{K_{1}(m x)}{x},
\end{aligned}
$$

where $x=\sqrt{\vec{x}^{2}+x_{4}^{2}}$ and $K_{1}$ is the McDonald function. In the massless limit, one obtains

$$
S_{0}(x) \rightarrow \frac{i \hat{x}}{2 \pi^{2} x^{4}}
$$

In the first order in $\sigma$, one obtains in the massless limit

$$
\begin{aligned}
S(0, T) & =S_{0}(0, T)+i \int d^{4} x S_{0}(0, x) \sigma|\vec{x}| S_{0}(x, T)+\cdots \\
& \equiv S_{0}(0, T)+S_{1}(0, T)+\cdots,
\end{aligned}
$$

where the function $S_{1}$ can be written in the massless limit as

$$
S_{1}(0, T)=\frac{i \sigma}{\left(2 \pi^{2}\right)^{2}} \int \frac{\hat{x}}{x^{4}}|\vec{x}| \frac{(\hat{x}-\hat{T})}{(x-T)^{4}} d^{4} x .
$$

Integration in Eq. (6) yields

$$
S_{1}(0, T)=\frac{i \sigma}{8 \pi T} .
$$

Consider now the higher-order terms in the expansion (5). The typical $O\left(\sigma^{n}\right)$ term looks like

$$
S_{n}(0, T)=i^{n} \int d^{4} x_{1} \cdots d^{4} x_{n} S_{0} \sigma\left|\vec{x}_{1}\right| S_{0} \cdots \sigma\left|\vec{x}_{n}\right| S_{0} .
$$

It is easy to see that the integrals are infrared-divergent at large $|\vec{x}|$ starting from the term with $n=2$, however for $m$ $\neq 0$ this divergence is eliminated and integrals are cut off by the mass at $x \sim 1 / m$. Therefore, typical $S_{n}(0, T)$ has the form, for $n>2$,

$$
S_{n}(0, T) \sim\left(\frac{\sigma}{m^{2}}\right)^{n} m^{3}
$$

while the $n=1$ term obtains corrections of the form

$$
S_{1}(0, T)=\frac{i \sigma}{8 \pi T}\left[m T K_{1}(m T)+O(m T)\right] .
$$

It is instructive to compare Eqs. (4), (7), and (9) with the nonrelativistic expansion (1). One can see that apart from the difference in free Green's functions, the first dynamical term is nonsingular in the nonrelativistic case (1), $G_{1}^{\text {osc }}$ $=-m \omega^{2} T / 12 \pi$, while it is singular in the relativistic case (7) if $T \rightarrow 0$.

To clarify the origin of this difference, one can compute $S_{1}(0, T)$ for the nonrelativistic Green's function with a linear potential. Note that the free Green's function in 3D is

$$
G_{0}^{\mathrm{nr}}\left(\vec{x}_{1}, t_{1} ; \vec{x}_{2}, t_{2}\right)=\left(\frac{m}{2 \pi\left(t_{2}-t_{1}\right)}\right)^{3 / 2} \exp \left(-\frac{m\left(\vec{x}_{2}-\vec{x}_{1}\right)^{2}}{2\left(t_{2}-t_{1}\right)}\right) \text {. }
$$

A calculation similar to Eq. (6) immediately yields

$$
G_{1}^{\mathrm{nr}}(0, T)=\frac{\sigma m}{8 \pi},
$$

which is nonsingular at small $T$ in contrast to $S_{1}(0, T)$ in Eq. (7). It is easy to see that also all higher terms in $\sigma^{n}$ are 
nonsingular due to the specific feature of the nonrelativistic Green's function (11): all time intervals are ordered $\left(t_{n}\right.$ $\left.>t_{n-1}>t_{n-2}\right)$ and all space intervals are cut off by the time intervals and the mass, so that a quark cannot escape far away during a short time interval, in contrast to the relativistic case, in which a light quark can travel as far as $1 / \mathrm{m}$ $\gg T$ for any small $T$. This crucial difference between nonrelativistic and relativistic dynamics causes the different behavior of the Green's functions at small distances or times.

\section{RELATIVISTIC EQUATION FOR THE HEAVY-LIGHT SYSTEM}

We shall discuss the situation for the field-theoretical model in this section, namely for the two-body system made of a spinor particle with mass $m$ and a heavy scalar antiparticle whose mass is considered infinite. We assume that this "meson" interacts with a confining gauge-field background, which is characterized by the Gaussian field strength correlator (see review [17] and references therein)

$$
\begin{aligned}
\Delta_{\mu_{1} \nu_{1}, \mu_{2} \nu_{2}}^{(2)}= & \left.\operatorname{tr}_{c}\left(F_{\mu_{1} \nu_{1}}\left(z_{1}\right) \Phi\left(z_{1}, z_{2}\right) F_{\mu_{2} \nu_{2}}\left(z_{2}\right) \Phi\left(z_{2}, z_{1}\right)\right)\right\rangle \\
= & \frac{1}{2}\left(\frac{\partial}{\partial z_{\mu_{1}}}\left(z_{\mu_{2}} \delta_{\nu_{1} \nu_{2}}-z_{\nu_{2}} \delta_{\nu_{1} \mu_{2}}\right)\right. \\
& \left.+\frac{\partial}{\partial z_{\nu_{1}}}\left(z_{\nu_{2}} \delta_{\mu_{1} \mu_{2}}-z_{\mu_{2}} \delta_{\mu_{1} \nu_{2}}\right)\right) D_{1}\left(z_{1}-z_{2}\right) \\
& +\left(\delta_{\mu_{1} \mu_{2}} \delta_{\nu_{1} \nu_{2}}-\delta_{\mu_{1} \nu_{2}} \delta_{\mu_{2} \nu_{1}}\right) D\left(z_{1}-z_{2}\right),
\end{aligned}
$$

where $\Phi(x, y)$ stays for the phase factor,

$$
\Phi(x, y)=\mathrm{P} \exp \left(i \int_{x}^{y} A_{\mu}(u) d u_{\mu}\right) .
$$

The Green's function of such a system can be represented as follows:

$$
\langle\bar{\psi}(x) \Phi(x, y) \psi(y)\rangle=S_{0}(x, y)+S_{2}(x, y)+\cdots,
$$

where $S_{0}$ is given by Eq. (3) while the first nontrivial interaction term has the form

$$
\operatorname{Tr} S_{2}(x, y)=\left\langle\int d^{4} u \int d^{4} w \operatorname{tr}\left(S_{0}(x, u) i \hat{A}(u) S_{0}(u, w) i \hat{A}(w) S_{0}(w, y)\right)\right\rangle
$$

where $\operatorname{tr}=\operatorname{tr}_{c} \operatorname{tr}_{L}$ is a product of traces over color and Lorentz indices. We adopt the Fock-Schwinger gauge condition with the base point $x_{0}=x: A_{\mu}(u)\left(u-x_{0}\right)_{\mu}=0$. In this gauge, the Green's function of the heavy particle gives some numerical factor whose exact form is inessential in what follows while the gauge field propagator takes the form

$$
\begin{aligned}
\left\langle\operatorname{tr}_{c} A_{\mu}(u) A_{\nu}(w)\right\rangle= & D(0) \cdot\left[(u-x)(w-x) \delta_{\mu \nu}\right. \\
& \left.-(u-x)_{\nu}(w-x)_{\mu}\right] \cdot f(u, w),
\end{aligned}
$$

where the dimensionless function $f(u, w)$ is given by the following expression:

$$
f(u, w)=\frac{1}{D(0)} \int_{0}^{1} d \alpha \alpha \int_{0}^{1} d \beta \beta D(\alpha u-\beta w) .
$$

Functions of this kind are often used in the formalism of coordinate gauges. One can find in the Appendix of the present paper a detailed analysis of $f(u, w)$ for a particular choice of Gaussian ansatz $D(z)=D(0) \exp \left(-z^{2} / T_{g}^{2}\right)$. We shall keep only the function $D(z)$ in what follows since the function $D_{1}(z)$ is not responsible for confinement effects. It was also found on the lattice that the nonperturbative part of $D_{1}(z)$ is significantly smaller than that of $D(z)$ in QCD; see [17] and references therein.

In momentum space, Eq. (16) takes the form

$$
\begin{aligned}
\operatorname{Tr} S_{2}(x, y)= & 4 i m \int \frac{d^{4} l}{(2 \pi)^{4}} \int \frac{d^{4} k}{(2 \pi)^{4}} \int_{0}^{1} \alpha d \alpha \int_{0}^{1} \beta d \beta \exp (i l(x-y)) \\
& \times \frac{D\left(k^{2}\right)}{l^{2}+m^{2}} \cdot\left[\frac{\partial}{\partial r_{\rho}} \frac{\partial}{\partial s_{\sigma}}\right]_{\substack{r=k \alpha \\
s=k \beta}}\left\{\frac{1}{(l-s+r)^{2}+m^{2}} \frac{1}{(l-s)^{2}+m^{2}} \times\left[\delta_{\rho \sigma}\left(3 m^{2}-l^{2}-2 l r-s r+s^{2}\right)+4 l_{\rho} l_{\sigma}-4 l_{\rho} s_{\sigma}\right.\right. \\
& \left.\left.+2 l_{\rho} r_{\sigma}-2 l_{\sigma} s_{\rho}+2 s_{\rho} s_{\sigma}-r_{\sigma} s_{\rho}-r_{\rho} s_{\sigma}\right]\right\} .
\end{aligned}
$$


The properties of the expression (19) are determined by the interplay of external parameters such as particle mass $m$ and distance $|x-y|$ and the properties of the confining background encoded in the function $D(z)$. In the case of QCD, the latter is usually computed in the course of lattice simulations [18]. It decays with distance and has some typical correlation length scale which we denote as $T_{g}$ throughout the paper. The exact dependence of $D(z)$ on $z$ is of no principal importance; one usually takes exponential fits (see [17]). At the origin, $D(z)$ is normalized to the nonperturbative gluon condensate according to

$$
D(0)=\frac{1}{12}\left\langle\operatorname{tr}_{c} F_{\mu \nu} F_{\mu \nu}\right\rangle .
$$

It is worth mentioning that the actual numerical value of $T_{g}$ in gluodynamics and QCD is rather small: it is estimated as $0.22 \mathrm{Fm}$ for quenched $\mathrm{SU}(3)$ and as $0.34 \mathrm{Fm}$ for full QCD with four flavors $[18,19,17]$. As will be clear from what follows, this circumstance bounds the region of applicability of conventional OPE based on local condensates.

We study first the heavy quark case, i.e., we assume that $m T_{g} \gg 1$. The integrals in Eqs. (16) and (19) are saturated at momenta $l^{2}$ of the order of the mass $m^{2}$ and one can make a systematic expansion over $1 / m T_{g}$. Straightforward although rather lengthy calculation leads to the following answer for the heavy quark condensate:

$$
\operatorname{Tr} S_{2}(x, x)=\frac{-i\left\langle\operatorname{tr}_{c} F_{\mu \nu} F_{\mu \nu}\right\rangle}{24 \pi^{2} m}\left[1-\frac{44}{45} \frac{1}{m^{2} \widetilde{T}_{g}^{2}}+O\left(\frac{1}{m^{4} \widetilde{T}_{g}^{4}}\right)\right],
$$

where $\widetilde{T}_{g}$ is defined as

$$
\frac{1}{\widetilde{T}_{g}^{2}}=\frac{1}{4 D(0)} \int \frac{d^{4} k}{\left(2 \pi^{4}\right)} D\left(k^{2}\right) k^{2}=\frac{\left\langle\operatorname{tr}_{c}\left(F D^{2} F\right)\right\rangle}{\left\langle\operatorname{tr}_{c} F^{2}\right\rangle},
$$

where the last relation is valid in the Gaussian approximation when all contributions from higher correlators are neglected. Let us mention that the virtuality of the quark condensate usually measured by the quantity

$$
\lambda_{q}^{2}=\frac{\left\langle\bar{\psi} D^{2} \psi\right\rangle}{\langle\bar{\psi} \psi\rangle}
$$

in the sum-rule approach is comparable with that of the gluon condensate (21); indeed, $\lambda_{q}^{2}=(0.4 \pm 0.1) \mathrm{GeV}^{2}$ according to [20], while $T_{g}$ was found on the lattice to be $(0.34$ $\pm 0.02) \mathrm{Fm}$ in $\mathrm{SU}(3)$ with four dynamical flavors [19], i.e., $\lambda_{q} \widetilde{T}_{g}$ is of the order of one. It could be instructive, therefore, to reconcile our approach with the method of nonlocal quark condensates worked out in [21,22] (see also [45]).

For $D(z) \propto \exp \left(-z^{2} / T_{g}^{2}\right)$ with the correlation length $T_{g}$ (as is used in the Appendix), one has $T_{g}=\sqrt{2} \widetilde{T}_{g}$. The first term in the expansion (20) is the well known OPE result for the heavy quark condensate [23]; see also [24]. The second term is the first nonlocal correction. It is worth mentioning that due to the smallness of $T_{g}$ (see above), it can be omitted as a numerically small correction for $b, t$ quarks only, while for $s, c$ quarks keeping only the first term in the expansion in $1 / m T_{g}$ is not to be considered as a good approximation.

Equations of the form (21) and (22) account for nonzero virtuality of vacuum lines in standard OPE language; one considers quantum averages, which contain derivatives. As we shall see in what follows, this language is not universal and implicitly assumes small averaged virtuality corresponding to the vacuum state, i.e., the large- $T_{g}$ limit. Another essential ingredient of this language is the use of equations of motion for such averages. Although it is rather easy to justify the validity of this component of the approach in the Abelian case, to the best of the author's knowledge this prescription has never been proved for non-Abelian theories with the level of rigor adopted in the field. Since we are discussing nonlocal correlators, the following remark is of importance. Consider the parallel transported field strength tensor $F_{\mu \nu}$, i.e.,

$$
G_{\mu \nu}\left(x, x_{0}\right)=\Phi\left(x_{0}, x\right) F_{\mu \nu}(x) \Phi\left(x, x_{0}\right),
$$

and the nonlocal gauge-invariant two-point correlator,

$$
\left\langle\operatorname{tr}_{c} G\left(x, x_{0}\right) G\left(y, x_{0}\right)\right\rangle .
$$

The above correlator depends on the positions of the points $x, y, x_{0}$ and on the profiles of the contours used in the factors $\Phi$. However, if $x \rightarrow y$, all these dependences disappear (phase factors cancel each other, while the $x$ dependence is prohibited by translational invariance), and the resulting local average coincides with $\left\langle\operatorname{tr}_{c} F^{2}\right\rangle$. Let us consider now an expansion of (23) if $|x-y|$ is small. In principle, one might consider two different expansions, with correlators involving derivatives in both cases. In the first case, it reads

$$
\begin{aligned}
\left\langle\operatorname{tr}_{c} G_{\mu \nu}\left(x, x_{0}\right) G_{\rho \sigma}\left(y, x_{0}\right)\right\rangle \approx & \left\langle\operatorname{tr}_{c} F_{\mu \nu} F_{\rho \sigma}\right\rangle \\
& +(y-x)_{\alpha} \cdot\left\langle\operatorname{tr}_{c} G_{\mu \nu}\left(x, x_{0}\right)\right. \\
& {\left.\left.\left[\frac{\partial G_{\rho \sigma}\left(y, x_{0}\right)}{\partial y_{\alpha}}\right]\right|_{y=x}\right\rangle+\cdots, }
\end{aligned}
$$

where the derivative is given by

$$
\begin{aligned}
\frac{\partial G_{\rho \sigma}\left(y, x_{0}\right)}{\partial y_{\alpha}}= & \Phi\left(x_{0}, y\right)\left(D_{\alpha} F_{\rho \sigma}(y)+i\left(y-x_{0}\right)_{\beta}\right. \\
& \left.\times \int_{0}^{1} s d s\left[G_{\beta \alpha}(z, y), F_{\rho \sigma}(y)\right]\right) \Phi\left(y, x_{0}\right)
\end{aligned}
$$

and $[\ldots, \ldots]$ in Eq. (25) denotes a commutator with respect to color indices. The second term (and all higher terms) on the right-hand side (rhs) of Eq. (24) contains a nonlocal part and depends on contour profiles and on the position of the points $x, x_{0}$ unless $x=y=x_{0}$. On the other hand, the expansion goes in powers of the quantity $(y-x)$, which is assumed to be small.

In the second case, one expands each $G$ in Eq. (23) in the vicinity of the point $x_{0}$ : 


$$
\begin{aligned}
\left\langle\operatorname{tr}_{c} G_{\mu \nu}\left(x, x_{0}\right) G_{\rho \sigma}\left(y, x_{0}\right)\right\rangle \approx & \left\langle\operatorname{tr}_{c}\left[F_{\mu \nu}\left(x_{0}\right)+\left(x-x_{0}\right)_{\alpha} D_{\alpha} F_{\mu \nu}\left(x_{0}\right)+\cdots\right]\left[F_{\rho \sigma}\left(x_{0}\right)+\left(y-x_{0}\right)_{\alpha} D_{\alpha} F_{\rho \sigma}\left(x_{0}\right)+\cdots\right]\right\rangle \\
\approx & \left\langle\operatorname{tr}_{c} F_{\mu \nu} F_{\rho \sigma}\right\rangle+\left(y-x_{0}\right)_{\alpha} \cdot\left\langle\operatorname{tr}_{c} F_{\mu \nu}\left(x_{0}\right) D_{\alpha} F_{\rho \sigma}\left(x_{0}\right)\right\rangle+\left(x-x_{0}\right)_{\alpha} \cdot\left\langle\operatorname{tr}_{c} D_{\alpha} F_{\mu \nu}\left(x_{0}\right) F_{\rho \sigma}\left(x_{0}\right)\right\rangle \\
& +\cdots .
\end{aligned}
$$

This is an expansion adopted in conventional OPE. In contrast with Eq. (24), nonlocal parts are absent. The price to pay, however, is that the expansion goes in $x-x_{0}, y-x_{0}$ instead of $y-x$. Needless to say, in many physical applications $|y-x|$ can be small whereas $\left|x-x_{0}\right|$ and $\left|y-x_{0}\right|$ are very large. Notice also that the opposite situation is impossible: smallness of $\left|x-x_{0}\right|,\left|y-x_{0}\right|$ implies smallness of $|y-x|$.

After this rather academic discussion we come back to the limit of small quark mass and/or correlation length $m T_{g} \ll 1$, which is opposite to what has been explored in Eq. (20). As was already mentioned, in real QCD, the parameter $\left\langle\operatorname{tr}_{c} F^{2}\right\rangle T_{g}^{4}$ can be considered as small, even in presence of dynamical quarks. In particular, typical momenta $l^{2}$ in Eq. (19) can be rather large in comparison with the nonperturbative scale given by the condensate $\sqrt{\left\langle\operatorname{tr}_{c} F^{2}\right\rangle}$ but still small when compared to the nonlocality scale $\sim T_{g}^{-2}$. The test particle resolves the nonlocality of vacuum field correlations in this regime.

The Green's function $S_{2}(x, y)$ in which we are interested is defined in Eq. (16). We are working in the coordinate representation here and choose the Fock-Schwinger gauge reference point $x_{0}$ at the origin $x_{0}=x=0$. We rewrite $S_{2}(x$ $=0, y$ ) using Eq. (17) as

$$
\begin{aligned}
S_{2}(0, y)= & \frac{i D(0)}{64 \pi^{6}} \int d^{4} u \int d^{4} w\left[\left(\frac{m}{u^{2}}-2 \frac{\hat{u}}{u^{4}}\right) \cdot[4(u w)-\hat{u} \hat{w}]\right. \\
& \times f(u, w) \cdot\left(2 \frac{\hat{u}-\hat{w}}{(u-w)^{4}}+\frac{m}{(u-w)^{2}}\right) \\
& \left.\times\left(2 \frac{\hat{w}-\hat{y}}{(w-y)^{4}}+\frac{m}{(w-y)^{2}}\right)\right]
\end{aligned}
$$

where we have kept in the propagators only those terms that are linear in mass $m$ since we consider the small-mass limit. The kernel $f(u, w)$ is defined in Eq. (18) and $\hat{u}=u_{\mu} \gamma_{\mu}$.

The actual value of this integral is defined by the properties of the function $f(u, w)$, which encodes all nonperturbative dynamics in the chosen Gaussian approximation. They are rather peculiar, however (see the Appendix), and this circumstance precludes one from obtaining an exact analytic answer. On the other hand, Eq. (27) can be calculated numerically for any particular ansatz for $D(x)$. Let us investigate the general structure of $S_{2}$. In the massless limit, one immediately obtains $\lim _{y \rightarrow 0} S_{2}(0, y)=0$ according to the absence of chiral symmetry breaking in the problem in question. It is seen that $S_{2}$ is UV-finite (small $u, w$ domain) because the nonperturbative background is soft: $\lim _{u, w \rightarrow 0} f(u, w)=\frac{1}{4}$. In the infrared domain $|u|,|w| \gg T_{g}$, the integral is convergent due to the properties of $f(u, w)$ (see the Appendix). One obtains in the massless case the following leading term at small $|y|$ :

$$
S_{2}(0, y)=-i c \cdot D(0) \cdot \hat{y}+O\left(y^{2}\right) \text {. }
$$

The numerical constant $c$ is determined by the function $f(u, w)$, but is $T_{g}$-independent. The massive parts of $S_{2}$ provide a finite contribution at $y=0$ :

$$
S_{2}(0, y) \sim i m D(0) T_{g}^{2} .
$$

If the mass is increasing and reaches values of the order of $T_{g}^{-1}$, it begins to work as an IR cutoff instead of $T_{g}$ and one comes back to Eq. (20). However, if the mass is small, then light quarks essentially feel the virtuality distribution of vacuum gluon fields [i.e., the profile of $f(u, w)$ ].

It is instructive to show how the potential problem considered in Sec. II appears from the field-theoretical framework invoked by us here. To this end, one should consider the equation for the heavy-light system which was obtained from the QCD Lagrangian in [14] in the limit of large $N_{c}$. Keeping only the Gaussian field correlator, one has instead of Eq. (2) the equation for the quark Green's function (made gauge-invariant due to the phase factor coming from the heavy source propagator),

$$
-i(\hat{\partial}+m) S(x, y)-i \int M(x, z) d^{4} z S(z, y)=\delta^{(4)}(x-y),
$$

where the nonlocal kernel $M(x, z)$ depends on the exact Green's function $S(x, z)$, making Eq. (28) nonlinear. Throughout this section, we are working in the so-called modified Fock-Schwinger gauge (see all details in [25]) where the temporal axis is singled out. We have retained for simplicity only the color electric part of the correlator as defined in [26], $\left\langle E_{i}(x) E_{k}(z)\right\rangle \sim \delta_{i k} D(x-z)$. Assuming for $D(x-z)$ the Gaussian ansatz, one arrives at the following form of the nonlocal kernel $M(x, y)$ :

$$
M(x, y)=D(0)(\vec{x} \vec{y}) f(\vec{x}, \vec{y}) S(x, y) \exp \left(-\frac{\left(x_{4}-y_{4}\right)^{2}}{T_{g}^{2}}\right),
$$

where $f(\vec{x}, \vec{y})$ is given in the Appendix. Notice that $\vec{x}, \vec{y}$ are three-dimensional vectors here and not four-dimensional as in Eq. (27).

As was shown in [15] using the relativistic WKB method developed in [14], the function $S(x, y)$ at large $\vec{x}, \vec{y}$, i.e., if $|\vec{x}|,|\vec{y}| \gg T_{g}$, can be written in the following form: 


$$
S(h, \vec{x}, \vec{y})=i e^{-(\sigma|\vec{x}|+m) h} g(\vec{x}, \vec{y})\left(\begin{array}{ll}
\theta(h) & \\
& \theta(-h)
\end{array}\right),
$$

where $h \equiv x_{4}-y_{4}$ and $g(x, y)$ is a smeared $\delta$ function,

$$
g(\vec{x}, \vec{y})=\widetilde{\delta}^{(3)}(\vec{x}-\vec{y}), \quad \sigma \vec{x}^{2} \sim \sigma \vec{y}^{2} \gg 1,
$$

moreover for large $\vec{x}$ and $\vec{y}$, and $|\vec{x}-\vec{y}| \ll|\vec{x}| \sim|\vec{y}|$ (see [14] and also the Appendix of the present paper),

$$
f(\vec{x}, \vec{y}) \sim \frac{T_{g}}{|\vec{x}|}
$$

and in this region one can integrate in Eq. (28) over $d^{4} z$, since

$$
M(x, z) \cong \sigma|\vec{x}| \delta^{(4)}(x-z),
$$

where the string tension $\sigma=(\pi / 2) D(0) T_{g}^{2}$ for the Gaussian ansatz [see Eq. (62) below]. Thus at large spacial arguments the kernel $M$ coincides with the linear potential considered in the previous section. Therefore, all estimates for terms in the expansion proportional to $M^{n}, n \geqslant 2$ are in agreement with those for the local case, Eq. (9), since integrals in these terms are essentially saturated by large spacial distances, $\left|\vec{x}^{(n)}\right|$ $\gg T_{g}$.

Although the potential behavior (33) is typical for the large- $T$ regime, it is instructive to show how the nonlocality cures the $1 / T$ behavior found in the local potential problem. We shall demonstrate now that the Green's functions in question have a finite limit when $T \rightarrow 0$ either for small or for large $T_{g}$. Let us briefly analyze the situation with the nonlocal equivalent of Eq. (6), i.e.,

$$
S_{1}^{(M)}(0, T)=\frac{i D(0)}{(2 \pi)^{2}} \int d^{4} x \int d^{4} y \frac{\hat{x}(\hat{y}-\hat{T})(\vec{x} \vec{y}) f(\vec{x}, \vec{y}) S(h, \vec{x}, \vec{y})}{x^{4}(y-T)^{4}} \exp \left(-\frac{h^{2}}{T_{g}^{2}}\right)
$$

with $S$ given in Eq. (30). It is convenient to introduce the dimensionless quantities

$$
\tilde{x}_{\mu}=x_{\mu} \sqrt{\sigma}, \quad \tilde{y}_{\mu}=y_{\mu} \sqrt{\sigma}, \quad \widetilde{T}_{g}=T_{g} \sqrt{\sigma}, \quad \widetilde{T}=T \sqrt{\sigma} .
$$

Rewriting Eq. (34) in terms of tilde variables, one immediately realizes that $S$ tends to the three-dimensional $\delta$ function only in the limit when $|\tilde{x}|,|\tilde{y}| \gg 1$ and otherwise the integral is defined by the region $|\tilde{x}| \sim|\tilde{y}| \sim 1$ when the nonlocality is at work, i.e., $|\tilde{x}-\tilde{y}| \sim|\tilde{x}|,|\tilde{y}|$. Imposing the limit of small $T_{g}$, i.e., $\widetilde{T}_{g} \ll 1$, one reduces two powers of $\tilde{x}, \tilde{y}$ in the numerator of Eq. (34), but the integral is still defined by large values of $\tilde{x}, \tilde{y}$ of the order of unity and one finally obtains

$$
S_{1}^{(M)}(0, T) \sim \text { const } \cdot \sigma^{3 / 2} .
$$

One can also show that the same estimate holds true for higher terms $O\left(m^{n}\right)$ and write $S_{n}^{(M)}(0, T) \sim c_{n} \sigma^{3 / 2}$. Consider now the opposite limit $\widetilde{T}_{g} \gg 1$, i.e., $T_{g} \gg 1 / \sqrt{\sigma}$. In this case, $\widetilde{T}_{g}$ does not confine the differences $\tilde{x}-\tilde{y}$ in $f(\tilde{x}, \tilde{y})$ and $\tilde{x}_{4}-\tilde{y}_{4}$ in the exponent in Eq. (34) to small values as compared to $|\tilde{x}|,\left|\tilde{x}_{4}\right|$ or $|\tilde{y}|,\left|\tilde{y}_{4}\right|$. Therefore, the integration over $d\left(\tilde{x}_{4}\right.$ $-\tilde{y}_{4}$ ) is limited only by the exponent in Eq. (30). As a result, one obtains for $S_{1}$ the following estimate (as always, we assume the mass $m$ to be not large, $m \lesssim \sqrt{\sigma}$ ):

$$
S_{1}^{(M)}(0, T) \sim \text { const } \cdot \frac{\sqrt{\sigma}}{T_{g}^{2}} .
$$

Thus in both cases the normal procedure of OPE, based on the analysis of subsequent terms of the perturbative expan- sion (and separating the soft and hard parts of the diagrams), is not applicable and one should sum up the whole series or else solve the nonlinear equation (28) exactly.

\section{ANOTHER FIELD-THEORETICAL EXAMPLE: HOW THE LINEAR CONFINEMENT IS BUILT UP OUT OF CONDENSATES}

In this section, we consider another example, namely a scalar (Higgs-type) particle interaction with the background Yang-Mills field, and again we calculate the heavy-light Green's function where the light particle is the color fundamental Higgs boson while the heavy source is like an antiquark. The Lagrangian and the Green's function are given by

$$
\begin{aligned}
& L=\frac{1}{4}\left(F_{\mu \nu}^{a}\right)^{2}+\left|D_{\mu} \varphi\right|^{2}-\frac{m^{2}|\varphi|^{2}}{2}, \\
& G^{(\varphi)}(0, T)=\langle\bar{\varphi}(0) \Phi(0, T) \varphi(T)\rangle .
\end{aligned}
$$

One can rewrite $G^{(\varphi)}$ as

$$
G^{(\varphi)}(0, T)=\left\langle\left(m^{2}-D_{\mu}^{2}\right)_{0, T}^{-1} \Phi(0, T)\right\rangle_{B}
$$

where we have introduced the external (background) field $B_{\mu}: D_{\mu}=\partial_{\mu}-i g B_{\mu}$. For simplicity of consideration, we shall confine ourselves to the vertices

$$
L_{4} \equiv g^{2}\left(B_{\mu}^{a b} \varphi^{b}\right)\left(B_{\mu}^{a c} \varphi^{c}\right)^{+}
$$

and choose the gauge [25] to write an equation for $G^{(\varphi)}(x, y)$ analogous to Eq. (28), 


$$
\left(m^{2}-\partial_{\mu}^{2}\right) G^{(\varphi)}(x, y)+\int I(x, z) G^{(\varphi)}(z, y) d^{4} z=\delta^{(4)}(x-y),
$$

where $I(x, z)=I^{(1)}(x, z)+I^{(2)}(x, z)$ and $I^{(1)}$ refers to the kernel with one power of $B_{\mu}$, while $I^{(2)}$ corresponds to the Lagrangian (40) and can be written as

$$
I^{(2)}(x, y)=\delta^{(4)}(x-y) g^{2} B_{\mu}^{2}(x) .
$$

The contributions from $I^{(1)}$ not analyzed by us here are of the same general structure as that of $I^{(2)}$ apart from nonlocality controlled by the particle mass $m$. Our consideration in this section is for illustration purposes only.

Let us take now the first-order graph in $g^{2}$ [keeping only $I^{(2)}$ in Eq. (41)]. In Euclidean space-time, one has

$$
G_{1}^{(\varphi)}(0, T)=g^{2}\left\langle\int d^{4} x G_{0}^{(\varphi)}(0, x) B_{\mu}^{2}(x) G_{0}^{(\varphi)}(x, T)\right\rangle_{B},
$$

where

$$
G_{0}^{(\varphi)}(x)=\frac{m}{4 \pi^{2} x} K_{1}(m x), \quad x=\sqrt{x^{2}} .
$$

We shall be interested in the vacuum-averaged expression for $G_{1}^{(\varphi)}$ and to this end one should express $B_{\mu}^{2}(x)$ in terms of field correlators (one way) or in terms of condensates (another standard way). In the gauge [25], one can write, e.g., for $B_{4}^{2}$

$$
\left\langle B_{4}^{2}(x)\right\rangle=\int_{0}^{x} d u_{\mu} \int_{0}^{x} d v_{\nu}\left\langle F_{\mu 4}(u) \Phi(u, v) F_{\nu 4}(v)\right\rangle
$$

and using [26], and keeping (as in Sec. III) only the confining part $D$, one has

$$
\left\langle B_{4}^{2}(x)\right\rangle=\int_{0}^{x} d u_{i} \int_{0}^{x} d v_{i} D(u-v), \quad i=1,2,3 .
$$

By way of example, let us consider an exponential ansatz for $D(u-v)$. As was already said, this behavior of $D(x)$ was observed in lattice simulations at distances larger than some typical correlation length $T_{g}$. So one has

$$
\left\langle B_{4}^{2}(x)\right\rangle=D(0) \vec{x}^{2} \int_{0}^{1} d \alpha \int_{0}^{1} d \beta \exp \left(\frac{|\vec{x}|}{T_{g}} \cdot|\alpha-\beta|\right) .
$$

Notice the absence of additional multipliers $\alpha, \beta$ in Eq. (47) contrary to Eq. (18). This is a property of the modified FockSchwinger gauge [25] (the temporal axis is singled out) used by us in this section, instead of the usual one used in Eq. (18) (one point is singled out).

Straightforward integration leads to the following result:

$$
\left\langle B_{4}^{2}(x)\right\rangle=2 D(0)|\vec{x}| T_{g}\left\{1-\frac{T_{g}}{|\vec{x}|} \cdot\left[1-\exp \left(-\frac{|\vec{x}|}{T_{g}}\right)\right]\right\} .
$$

At large $|\vec{x}| \gg T_{g}$, one has, from Eq. (48),

$$
\left\langle B_{4}^{2}(x)\right\rangle \approx 2 D(0) T_{g}\left(|\vec{x}|-T_{g}\right) .
$$

Notice that nonlocality enters Eq. (49) in an explicit way. At small $|\vec{x}|$, when $|\vec{x}| / T_{g} \sim 1$ the linear behavior of Eq. (49) is replaced by the quadratic one,

$$
\left\langle B_{4}^{2}(x)\right\rangle \approx D(0) \cdot \vec{x}^{2} .
$$

It is clear that the vacuum field correlator method implemented in Eqs. (45)-(49) demonstrates the creation of the string between the Higgs particle at the point $\left(\vec{x}, x_{4}\right)$ and the static source at the point $\left(0, x_{4}\right)$.

Now let us look at the same problem from the point of view of standard OPE. According to general rules [1,3-5], one should expand $F_{\mu 4}(u), F_{\nu 4}(v)$ in Eq. (45) in the vicinity of a point $\vec{u}=\vec{v}=0, u_{4}=v_{4}=x_{4}$ (in the Fock-Schwinger gauge, that would be the point $z_{0}$ usually chosen at the origin, $\left.z_{0}=0\right)$. In this way, one obtains

$$
\begin{aligned}
\left\langle B_{4}^{2}(x)\right\rangle= & \sum_{n, m} \frac{1}{(n+1) !(m+1) !} \\
& \times x_{i} x_{i_{1}} \cdots x_{i_{n}} x_{k} x_{k_{1}} \cdots x_{k_{m}} \\
& \times\left\langle D_{i_{1}} \cdots D_{i_{n}} F_{i 4}(0) D_{k_{1}} \cdots D_{k_{m}} F_{k 4}(0)\right\rangle .
\end{aligned}
$$

In this form (51), the appearance of the string is not visible, and one should rearrange the derivatives in a nontrivial way, so as to separate out the correlator $D(u-v)$ as in Eq. (46). Derivatives of the latter produce powers of $T_{g}^{-1}$, while a dependence on the sum $\frac{1}{2}\left(u_{i}+v_{i}\right)$ in the integral (46) is separated out to yield linear confinement in Eq. (49).

Now we consider the expansion of $G^{(\varphi)}$ in powers of $g^{2}$. From Eqs. (49), (43), and (44), it is clear that one obtains

$$
G_{1}^{(\varphi)}(0, T) \sim g^{2} \frac{c_{4}}{m}, \quad G_{n}^{(\varphi)} \sim g^{2 n}\left(\frac{c_{4}}{m^{3}}\right)^{n} m^{2},
$$

where the nonlocal constant $c_{4} \sim D(0) T_{g} \sim \int d z D(z)$. All integrals like Eq. (43) are diverging at large distances for $m$ $=0$ and cut off at $x \sim 1 / m$ when $m \neq 0$.

It is instructive to turn to the momentum space and define the following one-dimensional Fourier-transformed Green's function:

$$
G_{1}^{(\phi)}(Q)=\int_{-\infty}^{\infty} d T G_{1}^{(\phi)}(0, T) \exp (-i Q T) .
$$

[Our problem is $(3+1)$-dimensional; we do not perform a four-dimensional transformation, however, since the temporal axis was separated by our gauge choice from the begin- 
ning.] Since $\left\langle B_{4}^{2}(x)\right\rangle$ does not depend on the temporal coordinate $x_{4}$, integration in Eq. (43) is trivial:

$$
\begin{aligned}
G_{1}^{(\phi)}(M) & =\frac{g^{2}}{16 \pi^{2}} \int d^{3} x \frac{\exp (-2 M|\vec{x}|)}{\vec{x}^{2}}\left\langle B_{4}^{2}(|\vec{x}|)\right\rangle \\
& =\frac{g^{2}}{16 \pi} \frac{D(0)}{M^{3}} \cdot \frac{2 M T_{g}}{1+2 M T_{g}}
\end{aligned}
$$

where $M^{2}=Q^{2}+m^{2}$. Expression (54) has the following asymptotic expansions:

$$
\begin{aligned}
G_{1}^{(\phi)}(M)= & \frac{g^{2}}{16 \pi} \frac{D(0)}{M^{3}} \cdot\left[1-\frac{1}{2 M T_{g}}+\frac{1}{4 M^{2} T_{g}^{2}}\right. \\
& \left.+O\left(\frac{1}{M^{3} T_{g}^{3}}\right)\right], \quad M T_{g} \gtrsim 1
\end{aligned}
$$

and

$$
\begin{aligned}
G_{1}^{(\phi)}(M)= & \frac{g^{2}}{8 \pi} \frac{D(0) T_{g}}{M^{2}} \cdot\left[1-2 M T_{g}+4 M^{2} T_{g}^{2}\right. \\
& \left.+O\left(M^{3} T_{g}^{3}\right)\right], \quad M T_{g} \lesssim 1 .
\end{aligned}
$$

It is clearly seen that the actual answer is given by different series in regions $Q T_{g} \ll 1$ and $Q T_{g} \gg 1$ (we assume that $Q$ $\gg m$ and $M \approx Q$ ). The expansion (55) is associated with the standard OPE (51), while Eq. (56) goes essentially in powers of a nonlocal quantity. It is also worth mentioning that both expansions (55) and (56) are model-dependent beyond the leading condensate term, and the actual coefficients in Eqs. (55) and (56) are determined by the profile of $D(z)$.

\section{FEYNMAN-SCHWINGER FORMALISM AND OPE}

We start with the same Green's function $G^{(\varphi)}(0, T)$ and write the Feynman-Schwinger representation (FSR) for it (see $[27,28]$ where references to earlier papers are given),

$$
G^{(\varphi)}(0, T)=\int_{0}^{\infty} d s \int(\mathcal{D} z)_{0, T} \exp (-K)\left\langle W\left(C_{z}\right)\right\rangle .
$$

Here $K=m^{2} s+\frac{1}{4} \int_{0}^{s} \dot{z}_{\mu}^{2} d \tau, s$ is Schwinger proper time, and $\left\langle W\left(C_{z}\right)\right\rangle$ is a Wilson loop consisting of a straight line $(0, T)$ and the trajectory of the Higgs particle from 0 to $T$. Notice also that

$$
(\mathcal{D} z)_{0 T}=\prod_{n=1}^{n} \frac{d^{4} \Delta z(n)}{(4 \pi \varepsilon)^{2}} \int \frac{d^{4} p}{(2 \pi)^{4}} \exp \left[i p\left(\sum_{n} \Delta z(n)-T\right)\right] .
$$

In a Gaussian approximation, $\left\langle W\left(C_{z}\right)\right\rangle$ can be written as

$$
\begin{aligned}
\left\langle W\left(C_{z}\right)\right\rangle= & \exp \left(-\frac{g^{2}}{2} \int_{S} d \sigma_{\mu \nu}(u) \int_{S} d \sigma_{\rho \lambda}(v)\right. \\
& \left.\times\left\langle F_{\mu \nu}(u) F_{\rho \lambda}(v)\right\rangle\right)
\end{aligned}
$$

and we have omitted for simplicity the parallel transporters inside $\langle F F\rangle$. Here $S$ is the prescribed minimal area surface in the loop $C_{z}$ (there is no sensitivity on the choice of $S$ when all higher cumulants are kept; with the choice of the Gaussian correlators and minimal surface, the contribution of all higher correlators was estimated to be around a few percent; see [17] and references therein).

For small contour $C_{z}$ (which means that not only $T$ is small but also the spatial size of the contour is small), one has, from Eq. (59) [26],

$$
\left\langle W\left(C_{z}\right)\right\rangle=\exp \left(-\frac{g^{2} S^{2}}{24 N_{c}}\left\langle F_{\mu \nu}^{a}(0) F_{\mu \nu}^{a}(0)\right\rangle\right) .
$$

For a rectangular contour $C_{z}$ of an arbitrary size $R \times T$, one can write

$$
\langle W(C)\rangle=\exp \left(-\frac{1}{2} \int d^{2} x \int_{S} d^{2} y D(x-y)\right) .
$$

Choosing for simplicity $D(z)=D(0) \exp \left(-z^{2} / T_{g}^{2}\right)$, one has

$$
\sigma=\frac{1}{2} \int D(z) d^{2} z=\frac{\pi D(0)}{2} T_{g}^{2}
$$

and finally

$$
\langle W(C)\rangle=\exp \left[-\frac{\sigma R T}{\pi^{2}} L\left(\frac{T_{g}^{2}}{R^{2}}\right) L\left(\frac{T_{g}^{2}}{T^{2}}\right)\right],
$$

where we have defined

$$
L(u)=\int_{-\infty}^{\infty} d t e^{-t^{2} u} \frac{\sin ^{2} t}{t^{2}}
$$

with the expansions

$$
\begin{aligned}
& L(u)=\sqrt{\frac{\pi}{u}}\left\{1-\frac{11}{72 u^{2}}+\frac{1}{80 u^{4}}+\cdots\right\}, \quad u \gg 1, \\
& L(u)=\pi+O(\sqrt{u}), \quad u \ll 1 .
\end{aligned}
$$

Now we consider $\langle W\rangle$ inside the integral (57). If one assumes that for small $T$ one can indeed use the approximation of a small area of the loop $C_{z}$, i.e., Eq. (60), then one has in the relativistic case, but considering $T$ small, $T \ll 1 / m$, and expanding Eq. (60),

$$
G^{(\varphi)}(0, T)=G_{0}^{(\varphi)}(0, T)+G_{1}^{(\varphi)}(0, T)+\cdots,
$$

where 


$$
G_{1}^{(\varphi)}(0, T) \sim g^{2}\left\langle S^{2}\right\rangle\left\langle F^{a} F^{a}\right\rangle \frac{1}{T^{2}} \sim g^{2} T^{2}\left\langle F^{a} F^{a}\right\rangle .
$$

[We have assumed according to what was said before Eq. (60) that $\left\langle S^{2}\right\rangle \sim T^{4}$. This is a standard result of OPE analysis with a local condensate accompanied by higher powers of $T$ (or higher powers of $1 / Q^{2}$ in the momentum representation).]

Let us now consider again the term $O\left(g^{2}\right)$, but now taking into account the nonlocal character of the correlators $\langle F(x) F(y)\rangle$. To this end, we expand the Wilson loop in Eq. (57) and, making use of two simple identities

$$
\begin{gathered}
(\mathcal{D} z)_{x y}=(\mathcal{D} z)_{x u} d^{4} u(\mathcal{D} z)_{u v} d^{4} v(\mathcal{D} z)_{v y}, \\
\int_{0}^{\infty} d s \int_{0}^{s} d \tau_{1} \int_{0}^{\tau_{1}} d \tau_{2} f\left(s, \tau_{1}, \tau_{2}\right) \\
=\int_{0}^{\infty} d s \int_{0}^{\infty} d \tau_{1} \int_{0}^{\infty} d \tau_{2} f\left(s+\tau_{1}+\tau_{2}, \tau_{1}+\tau_{2}, \tau_{2}\right),
\end{gathered}
$$

one can write

$$
\begin{aligned}
G_{1}^{(\varphi)}(0, T)= & \int d^{4} x \int d^{4} y G_{0}^{(\varphi)}(0, x) \\
& \times\left\langle A_{\mu}(x) A_{\nu}(y)\right\rangle \dot{x}_{\mu} G_{0}^{(\varphi)}(x, y) \dot{y}_{\nu} G_{0}^{(\varphi)}(y, T) .
\end{aligned}
$$

The notation used in Eq. (70) implies that $\dot{x}_{\mu}(\tau)=d x_{\mu} / d \tau$, and $\left\langle A_{\mu}(x) A_{\nu}(y)\right\rangle$ is expressed through a vacuum average of field strength $\langle F(u) F(v)\rangle$, e.g., as in the coordinate gauge [25],

$$
\left\langle A_{4}(x) A_{4}(y)\right\rangle=\int_{0}^{x} d u_{i} \int_{0}^{y} d v_{k}\left\langle F_{i 4}(u) F_{k 4}(v)\right\rangle .
$$

One can show that $\dot{x}_{\mu} \rightarrow \stackrel{\leftrightarrow}{\partial} / \partial x_{\mu}$ and one recovers in Eq. (70) the usual perturbation expansion for $G^{(\varphi)}$, where now, in contrast to Sec. IV, only the linear vertices $\varphi^{2} A_{\mu} \overleftrightarrow{\partial}_{\mu}$ are taken into account [the term $\varphi^{2} A_{\mu}^{2}$ would also appear in Eq. (57) when one takes into account the term with $x=y$ ].

From Eq. (70), one can deduce that the rhs stays constant at large $|\vec{x}-\vec{y}|$ while it decreases for large $\left|x_{4}-y_{4}\right|$ [this is especially clear when one uses for the correlator $D(u-v)$ the Gaussian form]. Therefore, the integral (70) is convergent both at large $x, y$ and at small $x, y$. Integrating Eq. (70), one obtains

$$
G_{1}^{(\varphi)}(0, T) \sim T_{g}^{2}\left\langle g F^{a} g F^{a}\right\rangle \sim \sigma,
$$

since $D(0) \sim\left\langle g F^{a} g F^{a}\right\rangle \sim \sigma / T_{g}^{2}$. Thus one obtains a nonlocal constant for small $T \ll T_{g}$. Comparing Eqs. (68) and (72), one can see that at small $T$ the correct (nonlocal) procedure yields a larger (dominant) term as compared with the standard OPE estimation. The reason again lies in the fact that relativistic trajectories occupy a larger area for the Wilson loop when treated perturbatively and nonlocally, whereas in standard OPE treatment one attributes to this term the local condensate, implying that the Higgs particle (or quark) does not go far from the static source.

\section{REMARKS ON OPE IN ABELIAN THEORIES WITH CONFINEMENT}

We are going to discuss OPE in Abelian confining models in this section. The complications due to path ordering are absent in the Abelian case and one may consider a general expression for the two-point correlator of the field strengths in the form (13), where the functions $D(z), D_{1}(z)$ depend entirely on $z=x-y$. We assume that the confining properties of the theory are caused by the condensate of monopoles, hence the equations of motion take the form

$$
\partial_{\mu} F_{\mu \nu}=j_{\nu} ; \quad \partial_{\mu} \widetilde{F}_{\mu \nu}=J_{\nu},
$$

where $\widetilde{F}_{\mu \nu}=\frac{1}{2} \epsilon_{\mu \nu \rho \sigma} F_{\rho \sigma}$ and $j_{\mu}, J_{\mu}$ are electrically and magnetically charged currents, respectively. We define the polarization operator $\Pi\left(q^{2}\right)$ of the electric currents $j_{\mu}$ as

$$
\int d^{d} x\left\langle j_{\mu}(0) j_{\nu}(x)\right\rangle \exp (i q x)=\left(\delta_{\mu \nu} q^{2}-q_{\mu} q_{\nu}\right) \Pi\left(q^{2}\right) .
$$

Differentiating the Abelian analog of Eq. (13) and taking into account the equations of motion, it is straightforward to obtain the following relation:

$$
\Pi\left(q^{2}\right)=\int d^{d} x\left(D(x)+\frac{d}{2} D_{1}(x)+x^{2} \frac{d D_{1}}{d x^{2}}\right) \exp (i q x) .
$$

In the $d=4$ case, it can be rewritten in symmetric form as

$$
\begin{aligned}
& \left\langle j_{\mu}(0) j_{\nu}(x)\right\rangle+\left\langle J_{\mu}(0) J_{\nu}(x)\right\rangle \\
& \quad=-\frac{1}{6}\left(\partial^{2} \delta_{\mu \nu}-\partial_{\mu} \partial_{\nu}\right)\left\langle F_{\alpha \beta}(0) F_{\alpha \beta}(x)\right\rangle,
\end{aligned}
$$

where for the condensate one has

$$
\left\langle F_{\alpha \beta}(0) F_{\alpha \beta}(x)\right\rangle=6[D(x)+\widetilde{D}(x)] .
$$

The function $\widetilde{D}(x)=D(x)+2 D_{1}(x)+x^{2}\left(d D_{1} / d x^{2}\right)$ corresponds to the confining part of the correlator of dual field strengths $\widetilde{F}$ in the same way as $D(x)$ corresponds to the correlator of $F$ in Eq. (13). In the case of $d=4$ QED without monopoles, one easily finds [29]

$$
D(x) \equiv 0, \quad D_{1}(x)=\frac{1}{\pi^{2}}\left(\frac{e^{2}(x)}{x^{4}}-\frac{1}{x^{2}} \frac{d e^{2}\left(x^{2}\right)}{d x^{2}}\right) .
$$

For the polarization operator, one obtains

$$
\Pi\left(q^{2}\right)=-\frac{1}{\pi^{2}} \int d^{4} x \exp (i q x) \frac{d^{2} e^{2}\left(x^{2}\right)}{\left(d x^{2}\right)^{2}} .
$$

It is seen that the free field term $\sim e_{0}^{2} / x^{4}$ does not contribute to $\Pi\left(q^{2}\right)$ and the only nonzero contributions to the rhs of Eq. 
(75) come from either the running of the charge $e^{2}\left(x^{2}\right)$ (as in perturbation theory) or from nonperturbative parts of $D(x), D_{1}(x)$ if they are not equal to zero. This is easy to understand: in the absence of external currents, the theory describes free photons, vacuum is not polarizable, and charge is not running, so all current-current correlators must vanish identically. On the other hand, knowledge of the exact propagator (and hence $D$ and $D_{1}$ ) would be equivalent to knowledge of $\Pi\left(q^{2}\right)$.

Let us examine possible contributions to $\Pi\left(q^{2}\right)$ coming from the nonperturbative confining part of $\langle F F\rangle$ [i.e., from $D(x)]$ if it exists. Standard OPE reasoning would suggest looking for the leading term of this kind in the form $\left\langle F^{2}\right\rangle / q^{4}$. It is easy to see that for functions $D(x)$, which are smooth at the origin [for example, $D(x)=D(0) \exp \left(-x^{2} / T_{g}^{2}\right)$ ], the corresponding contribution to $\Pi\left(q^{2}\right)$ is exponentially suppressed at large $q^{2}$ (i.e., for $q^{2} T_{g}^{2} \gg 1$ ), which means that power corrections are absent in this case, i.e., the nonperturbative background is "too soft." In particular, there is no $D(0) / q^{4}$ term. For $D(x)$ such that it is not smooth but finite at the origin [e.g., for the often used exponential fit $D(x)$ $\left.=D(0) \exp \left(-|x| / T_{g}\right)\right]$, one has as a leading large- $q$ nonperturbative asymptotics

$$
\Delta \Pi\left(q^{2}\right) \sim \frac{D(0)}{T_{g}}\left(\frac{1}{q^{2}}\right)^{5 / 2} \sim \frac{\left\langle F^{2}\right\rangle}{T_{g} q^{5}} .
$$

The situation becomes even more dramatic if $D(x)$ is singular at the origin (as it happens, for example, in the London limit of the Abelian-Higgs model (AHM) [30]), where $D(x) \sim M^{2} / x^{2}$ if $x \rightarrow 0$. One has, in this particular case,

$$
\Delta \Pi\left(q^{2}\right)=\frac{M^{2}}{q^{2}+M^{2}} \propto \frac{M^{2}}{q^{2}} \text { then } q^{2} \gg M^{2} .
$$

This $1 / q^{2}$ regime in the AHM is bounded from above, however, by the Higgs boson mass $m_{H}$ : if $q^{2} \gtrsim m_{H}^{2}$, the Ginzburg-Landau description of the condensate is not valid, broken symmetry is restored, and microscopic degrees of freedom come into play. Presumably the same reasoning is applicable to the "thin" strings scenario proposed in [31]: at distances much smaller than coherence length, neither "thick" nor "thin" strings can be formed. Notice that the string tension $\sigma$ depends on $m_{H}$ logarithmically in the London limit: $\sigma \sim \ln \left(m_{H} / M\right)$.

An interesting problem of systematic construction of OPE in the Abelian-Higgs model is beyond the scope of the present paper [notice, in particular, that the equations of motion in the AHM are different from Eq. (73)]. Nevertheless, it is of interest to compare the result (81) with an answer for a massive photon propagator. ${ }^{1}$ It can be obtained from Eq. (78)

\footnotetext{
${ }^{1}$ We stress again that we are speaking only about the theory whose equations of motion are given by Eq. (73); therefore all effects such as confinement and/or mass gap are associated with complicated dynamics of the currents $J$.
}

taking $e^{2}(x)=m x K_{1}(m x)$, which corresponds to the massive vector field propagator $\left\langle A_{\mu}(0) A_{\nu}(x)\right\rangle$. Differentiation in Eq. (79) yields

$$
\Pi\left(q^{2}\right) \propto-\frac{m^{2}}{q^{2}} \quad \text { then } q^{2} \gg m^{2} .
$$

This result is obvious from the form of propagator in momentum space. Notice the sign difference between Eqs. (82) and (81). It can be said, following [32], that the leading power correction $\Delta \Pi\left(q^{2}\right)$ in confining theory is caused by an exchange of a massive particle with tachyonic mass. There is an obvious cancellation between Eqs. (81) and (82) in the AHM, since $M=m$ in the London limit in this theory, which again reflects the fact that free expressions for $D$ and $D_{1}$ have been taken (see above). To find genuine confining vacuum polarizability in the AHM requires explicit calculations along the lines of Sec. III and IV of the present paper and will be reported elsewhere.

\section{SPECTRAL REPRESENTATION OF GREEN'S FUNCTIONS AND OPE}

In this section, we shall look at OPE from another point of view, trying to calculate terms of OPE using the known properties of the spectrum of the gauge-invariant Green's function.

This type of analysis was done most extensively for the ' $t$ Hooft model $\left(1+1\right.$ QCD at large $\left.N_{c}\right)$ [33], where exact results for the spectra and Green's functions are known. (For details of the analysis, the reader is referred to [34-38].) We follow most closely the notation and the line of reasoning of [38]. We consider again the heavy-light system but now in the $d=1+1$ limit. The Green's function can be written as

$$
\begin{aligned}
G^{(Q q)}(x) & =\langle 0|\bar{q}(x) \Phi(x, 0) q(0)| 0\rangle \\
& =\sum_{n=0}^{\infty} \frac{1}{(2 n) !}\left\langle\bar{q}\left(x_{\mu} D_{\mu}\right)^{2 n} q\right\rangle .
\end{aligned}
$$

Defining, on the other hand, the correlation function

$$
\begin{aligned}
P\left(q^{2}\right) & =i \int e^{i q x} d^{2} x\langle 0|T\{\bar{q} Q(x), \bar{Q} q(0)\}| 0\rangle \\
& \sim i \int G^{(Q q)}(x) e^{i q x} d^{2} x,
\end{aligned}
$$

one can write an expansion in inverse powers of $E=m_{Q}$ $-q_{0}$,

$$
P(E)=\frac{1}{E}\left[\langle\bar{q} q\rangle-\frac{1}{E^{2}}\left\langle\bar{q} P_{0}^{4} q\right\rangle-\cdots\right]+\text { pert. part, }
$$

where $P_{0}=i D_{0}$.

On the other hand, one can write a spectral decomposition (dispersion relation) for $P(E)$, 


$$
P(E)=\frac{N_{c}}{2 \pi} m_{0} \sqrt{\pi} \sum_{n} \frac{f_{n}^{2}}{E+E_{n}} \sim \frac{N_{c}}{\pi} \sum_{n} \frac{1}{\sqrt{n}(\sqrt{n}+\varepsilon)},
$$

where we have used the notation $m_{0}^{2} \equiv g^{2} N_{c} / \pi, 2 m_{0} \sqrt{\pi} \varepsilon$ $=E$, and relations for the heavy-light spectrum [38]

$$
E_{n}=2 m_{0} \sqrt{\pi n}\left[1+O\left(\frac{\ln n}{n}\right)\right], \quad f_{n}^{2}=\sqrt{\pi} n\left[1+O\left(\frac{\ln n}{n}\right)\right] .
$$

Now one can compare Eqs. (85) and (86) and, expanding the latter in powers of $1 / E^{n} \sim 1 / \varepsilon^{n}$, one obtains [38] for the coefficients in Eq. (85)

$$
\left\langle\bar{q} P_{0}^{2 n} q\right\rangle \sim\langle\bar{q} q\rangle\left(\pi m_{0}\right)^{2 n} n !
$$

The factorial growth of the coefficients in Eq. (88) is typical both for $1+1$ and $3+1$, as will be shown below in this section.

One can do another derivation of the coefficients (88) starting from equations of motion, in which case instead of Eq. (88) one obtains

$$
\left\langle\bar{q}\left(x_{\mu} D_{\mu}\right)^{2 n} q\right\rangle \sim x^{2 n} n !\langle\bar{q} q\rangle\left(-\frac{g^{2}\langle\bar{q} q\rangle}{2 m_{q}}\right)^{n} .
$$

Thus another feature appears (or a puzzle, as it was formulated in [38]): condensates computed from the spectrum or from microscopic equations of motion have drastically different scales, $m_{0}^{2 n}$ in the first case and $\left(m_{0}^{3} / m_{q}\right)^{2 n}$ in the second case, where $m_{q}$ tends to zero in the chiral limit.

We shall now show that in the $3+1$ QCD, at least for $N_{c} \rightarrow \infty$ the situation is very similar to that of the 't Hooft model: (a) OPE coefficients of the $1 / Q^{2 n}$ expansion ("condensates") have factorially growing behavior; (b) Condensates calculated from the spectrum and from diagrams (plus equations of motion) are different.

Consider now the $3+1$ problem, a description of the selfenergy part $\Pi\left(q^{2}\right)$. For two light quarks, the standard OPE of $\Pi\left(Q^{2}\right)$ in the Euclidean region is well known [1]:

$$
\begin{aligned}
\Pi\left(Q^{2}\right)= & -\frac{1}{4 \pi^{2}}\left(1+\frac{\alpha_{s}}{\pi}\right) \ln \frac{Q^{2}}{\mu^{2}}+\frac{6 m^{2}}{Q^{2}}+\frac{2 m\langle\bar{q} q\rangle}{Q^{4}} \\
& +\frac{\alpha_{s}\langle F F\rangle}{12 \pi Q^{4}}+\cdots .
\end{aligned}
$$

Following [39], one can use the background perturbation theory for the calculation of $\Pi\left(Q^{2}\right)$ and represent it in the form

$$
\Pi\left(Q^{2}\right)=\Pi^{(0)}\left(Q^{2}\right)+\alpha_{s} \Pi^{(1)}\left(Q^{2}\right)+\alpha_{s}^{2} \Pi^{(2)}\left(Q^{2}\right) .
$$

Let us first consider $\Pi^{(0)}\left(Q^{2}\right)$ (for details of computations, the reader is referred to [39] and papers quoted therein).

In the large- $N_{c}$ limit, $\Pi^{(0)}\left(Q^{2}\right)$ has the form

$$
\Pi^{(0}\left(Q^{2}\right)=\frac{1}{12 \pi^{2}} \sum_{n=0}^{\infty} \frac{C_{n}}{Q^{2}+M_{n}^{2}} .
$$

The masses $M_{n}$ can be taken as the eigenvalues of the well-known Hamiltonian, which was derived from QCD with the assumption of area law for minimal surface and was shown to be valid for small angular momentum $L=0,1,2$ [40], while for larger $L$ the string rotation should be taken into account, $\Delta H_{\text {str }}$, yielding the correct Regge slope $(2 \pi \sigma)^{-1}$ for masses $M_{n}$ [40-42],

$$
H^{(0)} \Psi_{n}=M_{n} \psi_{n}, \quad H^{(0)}=2 \sqrt{\vec{p}^{2}+m_{f}^{2}}+\sigma r+\Delta H_{\mathrm{str}} .
$$

Solutions to Eq. (93) can be written in the form

$$
M_{n}^{2}=2 \pi \sigma\left(2 n_{r}+L\right)+M_{0}^{2},
$$

where $M_{0}^{2} \approx m_{\rho}^{2}$. For $C_{n}$, one has

$$
C_{n}(L=0)=\frac{2}{3} Q_{f}^{2} N_{c} m_{0}^{2}, \quad C_{n}(L=2)=\frac{1}{3} Q_{f}^{2} N_{c} m_{0}^{2} .
$$

Here $m_{0}^{2}=4 \pi \sigma$, and $Q_{f}$ is the electric charge of the quark of flavor $f$. Taking into account the degeneracy of masses with $L=0, n_{r}=1$ and $L=2, n_{r}=0$, the total $C_{n}$ is the sum

$$
\bar{C}_{n}=C_{n}(L=0)+C_{n}(L=2)=Q_{f}^{2} N_{c} m_{0}^{2} .
$$

Since $\bar{C}_{n}$ does not depend on $n$ in this approximation, one obtains the sum

$$
\sum_{n=0}^{\infty} \frac{1}{M_{n}^{2}+Q^{2}}=-\frac{1}{m_{0}^{2}} \psi\left(\frac{Q^{2}+M_{0}^{2}}{m_{0}^{2}}\right)+\text { const },
$$

where the constant term is divergent and is eliminated by renormalization of $\Pi\left(Q^{2}\right) \rightarrow \Pi\left(Q^{2}\right)-\Pi(0)$.

Here $\psi(z)$ is the Euler function,

$$
\psi(z)=\frac{\Gamma^{\prime}(z)}{\Gamma(z)},\left.\quad \psi(z)\right|_{z \rightarrow \infty}=\ln z-\frac{1}{2 z}-\sum_{k=1}^{\infty} \frac{B_{2 k}}{2 k z^{2 k}},
$$

where $B_{n}$ are Bernoulli numbers. Hence at large $Q^{2}$, the leading term in Eq. (98) yields

$$
\Pi^{(0)}\left(Q^{2}\right)=-\frac{Q_{f}^{2} N_{c}}{12 \pi^{2}} \ln \frac{Q^{2}+M_{0}^{2}}{\mu^{2}}+O\left(\frac{m_{0}^{2}}{Q^{2}}\right) .
$$

For $Q^{2} \gg M_{0}^{2}$, this term coincides with the leading term in the OPE (90) (the latter is written for $Q_{f}=1$ ).

From Eqs. (92) and (97), one can compute also the next terms of the expansion in $1 / Q^{2}$,

$$
\Pi^{(0)}\left(Q^{2}\right)=-\frac{Q_{f}^{2} N_{c}}{12 \pi^{2}} \ln \frac{Q^{2}+M_{0}^{2}}{\mu^{2}}+\sum_{n=1}^{\infty} \frac{\lambda_{2 n} m_{0}^{2 n}}{Q^{2 n}} .
$$


It is clear that $\lambda_{n}$ at large $n$ grow factorially due to the asymptotics of the Bernoulli numbers, $B_{2 n}$ $=\left[(-)^{n-1}(2 n) ! /\left(2^{2 n-1} \pi^{2 n}\right)\right] \zeta(2 n)$.

Two properties are clearly visible in the expansion (100): (a) the "condensates" $m_{0}^{2 n}$ are large, $m_{0} \approx 2.5 \mathrm{GeV}$, as compared to the standard OPE condensates, e.g., $\langle F F\rangle \sim 0.1$ $-0.2 \mathrm{GeV}^{4}$; (b) the coefficients $\lambda_{n}$ grow factorially, which is in agreement with the discussion in [36] and analysis of the 't Hooft model in $[37,38]$, signifying that the OPE series is asymptotic.

Thus in both cases $1+1$ and $3+1$, when confinement is present and the spectrum contains nondecreasing probabilities $C_{n}$ (which is a feature of the linear confining interaction), the OPE is a factorially diverging series, implying renormalon singularities in the Borel plane [39]. Another feature which is general to both $1+1$ and $3+1$ theories is the mismatch between condensates calculated via the spectrum [as in Eq. (100)] and via diagrammatic analysis [as in Eq. (90)]. In [38], a possible solution of this mismatch for the $1+1$ case was suggested, which introduces the notion of "effective condensates," which may differ from actual condensates (defined, for example, on the lattice) due to the asymptotic character of the OPE series.

In $3+1$ case, there is another possibility to explain the mismatch, namely one should take into account that coefficients $\lambda_{n}$ of all higher condensates get contributions not only from the leading terms in $n$ of $M_{n}$ and $c_{n}$ but also from the subleading terms, and the final result for, say, $\lambda_{4}$ could be two orders of magnitude smaller due to cancellation between different terms, thus removing the mismatch. However, this requires a mechanism of fine-tuning between the subleading coefficients, the physical reason for which is still not known.

One could leave the discussion of the mismatch at this point, if another check were not possible. Indeed, let us take the OPE with large (spectral) condensates and do a sum-rule analysis of experimental data for $e^{+} e^{-} \rightarrow$ hadrons with $I$ $=1$ (see [43]).

This analysis was done in [39] using the hadronic ratio $R^{I}(s)=12 \pi \operatorname{Im} \Pi^{I}(S)$. For $I=1$, adding the perturbative terms with the known coefficients as in [1,43], but taking the background modified coupling constant [39], e.g., in one loop

$$
\alpha_{B}\left(Q^{2}\right)=\frac{4 \pi}{b_{0} \ln \left(\frac{Q^{2}+M_{B}^{2}}{\Lambda_{B}^{2}}\right)},
$$

where $M_{B} \approx 1.5 \mathrm{GeV}$ and $\Lambda_{B}^{(3)} \approx 482 \mathrm{MeV}$, one has

$$
\begin{aligned}
R^{I=1}(s)= & \frac{3}{2} \sum_{n=0}^{\infty} C_{n}^{I=1} \delta\left(s-M_{n}^{2}\right) \\
& +\frac{3}{2}\left[1+\frac{\alpha_{B}(s)}{\pi}+1.64\left(\frac{\alpha_{B}}{\pi}\right)^{2}\right], \\
C_{n}^{I=1}= & m_{0}^{2}, \quad M_{n}^{2}=m_{\rho}^{2}+n m_{0}^{2}, \\
n & =1,2, \ldots ; \quad C_{0}=\frac{2}{3} m_{0}^{2},
\end{aligned}
$$

and the corresponding Borel transform is

$$
\tilde{I}_{0}(M)=\frac{2}{3 M^{2}} \int_{0}^{\infty} d s e^{-s / M^{2}} R^{I=1}(s) .
$$

Substituting Eqs. (101) into Eq. (102) yields

$$
\begin{aligned}
\widetilde{I}_{0}(M)= & \frac{m_{0}^{2}}{M^{2}}\left\{\frac{2}{3} e^{-m_{\rho}^{2} / M^{2}}+\sum_{n=1}^{\infty} e^{-\left(m_{\rho}^{2}+n m_{0}^{2}\right) / M^{2}}\right\}+\frac{\alpha_{B}(M)}{\pi} \\
& +2.94\left(\frac{\alpha_{B}(M)}{\pi}\right)^{2}, \quad m_{0}^{2}=4 \pi \sigma .
\end{aligned}
$$

This should be compared to the standard result [1] with standard (small) condensates,

$$
\begin{aligned}
\widetilde{I}_{0}^{\mathrm{st}}(M)= & +\frac{\alpha_{s}(M)}{\pi}+2.94\left(\frac{\alpha_{s}(M)}{\pi}\right)+\frac{\pi^{2}}{3} \frac{G_{2}}{M^{4}} \\
& +\frac{448 \pi^{3} \alpha_{s}}{81} \frac{|\langle 0|\bar{q} q| 0\rangle|^{2}}{M^{6}} .
\end{aligned}
$$

In Eq. (104), $\alpha_{s}(M)$ is standard, i.e., obtained from $\alpha_{B}$ by setting $M_{B} \equiv 0$.

It is clear that Eq. (103) contains in the Borel plane a set of poles at $M^{2}=M_{k}^{2}= \pm i\left(m_{0}^{2} / 2 \pi k\right), k=1,2, \ldots$ and an essential singularity at $M=0$. These features imply the presence of renormalons and are connected to the factorial growth of coefficients $\lambda_{2 n}$ in Eq. (100).

Now remarkably both Borel transforms lie inside the corridor of experimental errors, thus describing satisfactorily data with very different values of condensates (for details of comparison, see [39]). This situation is becoming even more unclear: not only does one have two sets of condensates (and consequently two sets of sum rules), but in addition experimental data cannot give preference to either of them.

While leading perturbative large- $M$ asymptotics of $\widetilde{I}_{0}(M)$ and $\widetilde{I}_{0}^{\text {st }}(M)$ coincide, there is an important difference at small $M$ : while $M^{2} \widetilde{I}_{0}(M)$ is defined for all $M, M^{2} \widetilde{I}_{0}^{\text {st }}(M)$ is diverging for $M \rightarrow 0$ due to higher condensates and higher powers of $\alpha_{s}(M)$.

\section{CONCLUSIONS AND OUTLOOK}

The main emphasis of the present paper is the influence of confinement on the behavior of Green's functions in their dependence on momentum and the behavior of Borel transforms. We stressed above everywhere the importance of large distances working in the coordinate representation, especially for light quarks in the presence of confinement. As the first and most clear example, the Green's function of the Dirac equation with a linear scalar potential was considered and it was demonstrated that the Euclidean time expansion (the equivalent of the Borel transform for heavy-light systems) looks completely different from the nonrelativistic case and from the template oscillator Green's function. In this way, it was shown that large distances may be important 
even for small Euclidean times and may bring about new terms in the OPE in coordinate space.

As another example, we have treated the nonlinear equations for a quark in the heavy-light system-the nonlocal equivalent of the Dirac case - and found that again the result is different from what one would expect in standard OPE, but the terms of expansion turn out to be constant, $S_{1}(T)$ $\sim$ const $\cdot \sigma^{3 / 2}$. Translating this contribution into the form of the usual correlation function $\Pi\left(Q^{2}\right)$ of vector currents (as it is done in the reaction $e^{+} e^{-} \rightarrow$ hadrons), one would have the contribution $\Delta \Pi\left(Q^{2}\right) \sim m \sigma^{3 / 2} / Q^{4}$, which is similar to the standard term $m\langle\bar{q} q\rangle / Q^{4}$ and is presumably one term in the subseries generating $m\langle\bar{q} q\rangle$. In this example, large distances, explicitly accounted for in our analysis, do not produce new OPE terms but give some path to calculating the chiral condensate through confinement characteristics (i.e., string tension $\sigma$ ). One arrives at similar conclusions studying the Green's functions in the Feynman-Schwinger formalism; see Sec. V.

In Sec. IV, in contrast to that, another problem was elucidated: how is linear confinement built up out of higher condensates of OPE? The answer is given by a comparison of Eqs. (46)-(49) and (51). Indeed, the infinite sum of derivatives of field correlators in Eq. (51) is equivalent to the linear confinement term in Eq. (49), and to extract it explicitly one needs to rearrange all derivatives.

We have briefly analyzed Abelian electrodynamics with monopoles in Sec. VI and described different possible sources of nonstandard OPE terms, e.g., $1 / q^{2}$. To investigate OPE in Abelian models with confinement, notably in the AHM systematically, is an interesting task for the future.

Finally, the last problem considered in the paper concerns the derivation of OPE from the spectral representation of the meson Green function. When the spectrum and coefficients $c_{n}$ (equivalent to the quark decay constants $f_{\pi}$ ) are known, the OPE is calculated automatically and can be compared with that obtained "microscopically," i.e., via Feynman diagrams in the external fields and equations of motion.

In the $d=1+1 \mathrm{QCD}$, this program was fully investigated in a series of papers (see, e.g., [38] and references therein) and a mismatch between condensates obtained in those two ways was found.

In the $d=3+1 \mathrm{QCD}$, the situation is similar, and as shown in [39] and in the present paper, the mismatch of condensates in scales and order of magnitude is also evident. The situation is sharpened by the fact that the QCD sum rules for $e^{+} e^{-} \rightarrow$ hadrons reproduce experimental data for both choices of condensates.

We have not tried here to resolve this puzzle, and leave it for the future. There are two important topics in OPE we have not discussed. First, this is the partial summation of the OPE terms which can be done by introduction of nonlocal condensates in OPE, initiated and studied in [21,22]. It would be interesting to find the link between our treatment of long-distance nonperturbative physics and the method of nonlocal condensates worked out in $[21,22]$. Second, the problem of perturbative-nonperturbative interference, which may produce new singular OPE terms, such as $1 / q^{2}$, which was discussed in $[32,44,8]$, is touched upon in Sec. VI only briefly. This set of problems certainly deserves further study.

\section{ACKNOWLEDGMENTS}

The authors acknowledge support from Grants No. RFFI00-02-17836, No. RFFI-00-15-96786, and No. INTAS 00110. V.S. acknowledges support from the foundation "Fundamenteel Onderzoek der Materie" (FOM), which is financially supported by the Dutch National Science Foundation (NWO). V.S. also acknowledges support from Grant No. RFFI-01-02-06284. Yu.S. acknowledges financial support from INTAS Grant No. 00-00366.

\section{APPENDIX}

We discuss in this appendix the properties of the kernel (18), which we used in the main text. The reader is referred to Appendix 3 of the paper [14] where the 3D counterpart of Eq. (18) was analyzed. Since the kernels of the form (18) play an important role in the discussed formalism, we present an independent detailed analysis here both for possible future applications and for the reader's convenience.

We are interested in the properties of the following function:

$$
f(\vec{\eta}, \vec{\rho})=\int_{0}^{1} d \alpha \alpha \int_{0}^{1} d \beta \beta \exp \left(-\frac{(\alpha \vec{\eta}-\beta \vec{\rho})^{2}}{T_{g}^{2}}\right),
$$

where $\vec{\eta}, \vec{\rho}$ are $d$-dimensional vectors with angle $\theta$ between them. We denote absolute values of the arguments as $\eta$ $=|\vec{\eta}|, \rho=|\vec{\rho}|$, and we assume in what follows, without loss of generality, that $\eta \geqslant \rho$. The symmetry of formulas below with respect to the exchange $\rho \leftrightarrow \eta$ [which is manifest in the definition (A1)] is to be restored by replacements $\rho$ $\rightarrow \min \{\rho, \eta\}$ and $\eta \rightarrow \max \{\rho, \eta\}$.

It is instructive to consider four different asymptotic regions:

(i) $\eta, \rho \sim T_{g}$; (ii) $\eta \gg T_{g} ; \rho \sim T_{g}$; (iii) $\eta, \rho \gg T_{g} ; \theta \gtrsim 1$; (iv) $\eta, \rho \gg T_{g} ; \theta \ll 1$

In the region (i), one can expand Eq. (A1) in Taylor series with respect to both arguments. Subsequent integration is straightforward,

$$
\begin{aligned}
f(\vec{\eta}, \vec{\rho})= & \frac{1}{4}-\frac{\rho^{2}+\eta^{2}}{8 T_{g}^{2}}+\frac{2 \rho \eta \cos \theta}{9 T_{g}^{2}}+\frac{\rho^{4}+\eta^{4}}{24 T_{g}^{4}} \\
& +\frac{\rho^{2} \eta^{2}}{8 T_{g}^{2}}\left(\cos ^{2} \theta+\frac{1}{2}\right)-\frac{2 \rho \eta \cos \theta}{15 T_{g}^{4}} \\
& \times\left(\rho^{2}+\eta^{2}\right)+O\left(T_{g}^{-6}\right) .
\end{aligned}
$$

In derivation of expression (20) in the main text, we have used in fact the leading term of this asymptotics (i.e., $\frac{1}{4}$ ).

In the regions (ii), (iii), and (iv), we will systematically omit exponentially small terms, i.e., terms proportional to 
$\exp \left(-\eta^{2} / T_{g}^{2}\right)$ and also terms $\sim \exp \left(-\rho^{2} / T_{g}^{2}\right)$ in the regions (iii) and (iv). One easily obtains the following expression in the region (ii):

$$
\begin{aligned}
f(\vec{\eta}, \vec{\rho})= & \frac{T_{g}^{2}}{\eta^{2}}\left(\frac{1}{4}+\frac{\sqrt{\pi}}{6} \frac{\rho \cos \theta}{T_{g}}+\frac{1}{8} \frac{\rho^{2}}{T_{g}^{2}}\left(2 \cos ^{2} \theta-1\right)\right. \\
& \left.-\frac{\sqrt{\pi}}{10} \frac{\rho^{3} \cos \theta \sin ^{2} \theta}{T_{g}^{3}}+O\left(\rho^{4}\right)\right)
\end{aligned}
$$

Now we come to the regions (iii) and (iv). It is instructive to introduce the following variables:

$$
\begin{aligned}
& s=\frac{(\vec{\eta} \rho-\vec{\rho} \eta)^{2}}{T_{g}^{2}}=\frac{4 \eta^{2} \rho^{2}}{T_{g}^{2}} \sin ^{2} \frac{\theta}{2}, \\
& q=\frac{(\vec{\eta} \rho+\vec{\rho} \eta)^{2}}{4 T_{g}^{2}}=\frac{\eta^{2} \rho^{2}}{T_{g}^{2}} \cos ^{2} \frac{\theta}{2},
\end{aligned}
$$

and also $\xi=\sqrt{s} / \sqrt{q}=2 \tan (\theta / 2)$.

In the region (iii), the upper limit of the integration in Eq. (A1) can be shifted to infinity up to exponentially small corrections. The function $f(\vec{\eta}, \vec{\rho})$ in the region (iii) can be written, therefore, as

$$
f(\vec{\eta}, \vec{\rho})=\frac{T_{g}^{4}}{4 \eta^{2} \rho^{2}} \cdot \phi(\xi)
$$

where $\phi(\xi)$ is given by

$$
\begin{aligned}
\phi(\xi)= & \frac{\sqrt{\pi}}{8} \frac{\left(4+\xi^{2}\right)^{2}}{\xi^{3}} \int_{0}^{\infty} d y \exp \left(-y^{2}\right)\left\{\left[1-\operatorname{erf}\left(\frac{y \xi}{2}\right)\right]\right. \\
& \left.\times\left(1-\frac{y^{2} \xi^{2}}{2}\right)+\frac{y \xi}{\sqrt{\pi}} \exp \left(-\frac{y^{2} \xi^{2}}{4}\right)\right\}
\end{aligned}
$$

The function $\phi(\xi)$ is a monotonically decreasing function of $\xi$. When $\xi$ is going to infinity, $\phi(\xi)$ is approaching the following asymptotics:

$$
\phi(\xi)=\frac{1}{3}+\frac{32}{15} \frac{1}{\xi^{2}}+O\left(\xi^{-4}\right) .
$$

At the point $\xi=2$, which corresponds to $\theta=\pi / 2$ and hence orthogonal vectors $\vec{\eta}$ and $\vec{\rho}$, one finds $\phi(2)=1$, in agreement with simple direct calculation from Eq. (A1).

We are now in the position to analyze the properties of $f(\vec{\eta}, \vec{\rho})$ in the region (iv), where $\xi \sim \theta \ll 1$. Making the change of variables, one gets from Eq. (A1)

$$
\begin{aligned}
f(\vec{\eta}, \vec{\rho})= & -\frac{T_{g}^{2}}{\sin \theta}\left[\left\{\int_{\sqrt{s} / \eta \xi}^{0} d y \int_{y \xi / 2}^{(\sqrt{s} / \eta)-(y \xi / 2)} d x\right.\right. \\
& +\int_{0}^{-\sqrt{s} / \rho \xi} d y \int_{-y \xi / 2}^{(\sqrt{s} / \rho)+(y \xi / 2)} d x \\
& +\int_{0}^{-w} d y \int_{-y \xi / 2}^{(\sqrt{s} / \eta)-(y \xi / 2)} d x \\
& \left.-\int_{0}^{-w} d y \int_{-y \xi / 2}^{(\sqrt{s} / \rho)+(y \xi / 2)} d x\right\}\left(\frac{x^{2}}{s}-\frac{y^{2} \xi^{2}}{4 s}\right) \\
& \left.\times \exp \left(-x^{2}-y^{2}\right)\right],
\end{aligned}
$$

where $w=\sqrt{q}(1 / \rho-1 / \eta)=(\eta-\rho) \cos (\theta / 2) / T_{g}$. One can rewrite Eq. (A8) in the following form:

$$
\begin{aligned}
f(\vec{\eta}, \vec{\rho})= & \frac{T_{g}^{2}}{\sin \theta} \frac{1}{s}\left[g\left(\frac{\sqrt{s}}{\eta}, \xi\right)+g\left(\frac{\sqrt{s}}{\rho}, \xi\right)+f_{2}\left(\frac{\sqrt{s}}{\eta}, w, \xi\right)\right. \\
& \left.-f_{2}\left(\frac{\sqrt{s}}{\rho}, w,-\xi\right)\right]
\end{aligned}
$$

where the $\xi$ expansion of the functions $g, f_{3}, f_{4}$ can be performed systematically. It gives

$$
\begin{aligned}
g(z, \xi)= & \frac{\pi}{8} \kappa(z)-\frac{\exp \left(-z^{2}\right) z^{2}}{4} \cdot \xi \\
& -\frac{\pi}{32}\left(\kappa(z)+\frac{2 z^{3}}{\sqrt{\pi}} \exp \left(-z^{2}\right)\right) \cdot \xi^{2}+O\left(\xi^{3}\right) \\
f_{2}(z, w, \xi)= & \operatorname{erf}(w) \frac{\pi}{8} \kappa(z)+\left[1-\exp \left(-w^{2}\right)\right] \frac{\exp \left(-z^{2}\right) z^{2}}{4} \cdot \xi \\
& +O\left(\xi^{2}\right),
\end{aligned}
$$

where the function $\kappa(z)$ is defined as

$$
\kappa(z)=\operatorname{erf}(z)-\frac{2 z}{\sqrt{\pi}} \exp \left(-z^{2}\right)
$$

Extracting coefficient functions in front of higher powers in $\xi$ is a matter of straightforward algebra.

The expressions (A10) and (A11) are exact at the given order in $\xi$ up to omitted exponentially small terms. They can be simplified in different limiting cases. If $w=0$ (i.e., $\eta$ $=\rho$ ), one has $f_{2}=0$, while the first two terms on the rhs of Eq. (A9) are equal. In the opposite limit $w \rightarrow \infty$, the following relations hold true: 


$$
\begin{gathered}
\lim _{w \rightarrow \infty}\left[g\left(\frac{\sqrt{s}}{\rho}, \xi\right)-f_{2}\left(\frac{\sqrt{s}}{\rho}, w,-\xi\right)\right]=0, \\
\lim _{w \rightarrow \infty} f_{2}\left(\frac{\sqrt{s}}{\eta}, w, \xi\right)=g\left(\frac{\sqrt{s}}{\eta},-\xi\right) .
\end{gathered}
$$

Notice that in all cases the first argument of the functions $g, f_{2}$ need not be small: $\sqrt{s} / \eta=\left(2 \rho / T_{g}\right) \sin (\theta / 2)$ and in the region (iv) $\rho \gg T_{g}$, but $\theta \ll 1$.

In terms of the original variables $\eta, \rho, \theta$, the leading term in Eq. (A9) can be represented as

$$
\begin{aligned}
f(\vec{\eta}, \vec{\rho}) \approx & \frac{\pi}{64 \sin ^{3} \frac{\theta}{2} \cos \frac{\theta}{2}} \frac{T_{g}^{4}}{\eta^{2} \rho^{2}}\left[\kappa\left(\frac{2 \rho \sin \frac{\theta}{2}}{T_{g}}\right)[1+\operatorname{erf}(w)]\right. \\
& \left.+\kappa\left(\frac{2 \eta \sin \frac{\theta}{2}}{T_{g}}\right)[1-\operatorname{erf}(w)]\right]
\end{aligned}
$$

where $w=(\eta-\rho) \cos (\theta / 2) / T_{g}$ and $\kappa(z)$ is defined in Eq. (A12), $\kappa(z)>0$ if $z>0$. This expression is valid in the small$\theta$ limit.

Notice that $f$ is nonsingular if $\theta \rightarrow 0$ [which is evident from Eq. (A1)],

$$
\lim _{\theta \rightarrow 0} f(\vec{\eta}, \vec{\rho})=\frac{T_{g} \sqrt{\pi}}{6}\left[\frac{\rho}{\eta^{2}}+\frac{\eta}{\rho^{2}}+\operatorname{Erf}\left(\frac{\eta-\rho}{T_{g}}\right)\left(\frac{\rho}{\eta^{2}}-\frac{\eta}{\rho^{2}}\right)\right]
$$

One needs some simple extrapolating representation of Eq. (A1) for practical calculations. Notice that it is only asymptotic behavior of $f(\vec{\eta}, \vec{\rho})$ that matters; the particular form of Gaussian kernel was taken in Eq. (A1) just as an example. A possible expression respecting all desired properties of $f$ in the regions of large $\eta, \rho$ is as follows:

$$
f(\vec{\eta}, \vec{\rho}) \approx \frac{T_{g}^{4}}{4 \eta^{2} \rho^{2}} l(\theta),
$$

where the function $l(\theta)$ has the following "focusing" property: being integrated with a regular function $F(\theta)$, it acts like a smoothed $\delta$ function (see [14]),

$$
\int d \theta F(\theta) l(\theta) \approx c_{1} \frac{\rho^{3}}{T_{g}^{3}} F\left(\frac{c_{0} T_{g}}{\rho}\right)+c_{2} \int d \theta F(\theta),
$$

where $c_{0}, c_{1}, c_{2}$ are some constants of the order of unity. It is worth remembering that $\rho$ is the length of the smaller vector in our notation, i.e., $\rho=\min \{\rho, \eta\}$. In particular, it is seen that in the limit of large $\rho \gg T_{g}$, the small $\theta$ asymptotics gives a dominant contribution unless $F(\theta)$ vanishes at the origin faster than $\theta^{3}$.
[1] M.A. Shifman, A.I. Vainshtein, and V.I. Zakharov, Nucl. Phys. B147, 385 (1979).

[2] K. Wilson, Phys. Rev. 179, 1499 (1969); K. Symanzik, Commun. Math. Phys. 23, 49 (1971); C. Callan, Phys. Rev. D 5, 3302 (1972); R. Brandt, Fortschr. Phys. 18, 249 (1970).

[3] S. Narison, QCD Spectral Sum Rules (World Scientific, Singapore, 1989).

[4] Vacuum Structure and QCD Sum Rules, edited by M.A. Shifman (North-Holland, Amsterdam, 1992).

[5] A.V. Radyushkin, hep-ph/0101227.

[6] V.A. Novikov, M.A. Shifman, A.I. Vainshtein, and V.I. Zakharov, Nucl. Phys. B191, 301 (1981).

[7] V.I. Zakharov, "The 1999 Sakurai Prize Lecture," hep-ph/9906264.

[8] K.G. Chetyrkin, S. Narison, and V.I. Zakharov, Nucl. Phys. B550, 353 (1999).

[9] M.A. Shifman, Annu. Rev. Nucl. Part. Sci. 33, 199 (1983).

[10] U. Marquard and H.G. Dosch, Phys. Rev. D 35, 2238 (1987).

[11] H.G. Dosch and U. Lisenfeld, Phys. Lett. B 219, 493 (1989).

[12] H.G. Dosch and Yu.A. Simonov, Z. Phys. C 45, 147 (1989).

[13] V.A. Novikov, M.A. Shifman, A.I. Vainshtein, and V.I. Zakharov, Fortschr. Phys. 32, 11 (1984).

[14] Yu.A. Simonov, Phys. At. Nucl. 60, 2069 (1997).

[15] Yu.A. Simonov and J.A. Tjon, Phys. Rev. D 62, 014501 (2000).

[16] Yu.A. Simonov and J.A. Tjon, Phys. Rev. D 62, 094511 (2000).
[17] A. Di Giacomo, H.G. Dosch, V. Shevchenko, and Yu. Simonov, hep-ph/0007223.

[18] M. Campostrini, A. Di Giacomo, and G. Mussardo, Z. Phys. C 25, 173 (1984); M. Campostrini, A. Di Giacomo, and S. Olejnik, ibid. 34, 577 (1986).

[19] A. Di Giacomo, E. Meggiolaro, and H. Panagopoulos, Nucl. Phys. B483, 371 (1997)

[20] V.M. Belyaev and B.L. Ioffe, Zh. Eksp. Teor. Fiz. 83, 876 (1982); A.A. Ovchinnikov and A.A. Pivovarov, Yad. Fiz. 48, 1135 (1988); M. Kremer and G. Schierholz, Phys. Lett. B 194, 283 (1987).

[21] S.V. Mikhailov and A.V. Radyuskin, JETP Lett. 43, 712 (1986); Sov. J. Nucl. Phys. 49, 494 (1989).

[22] A.P. Bakulev and A.V. Radyuskin, Phys. Lett. B 271, 223 (1991); S.V. Mikhailov, Sov. J. Nucl. Phys. 56, 143 (1993); A.P. Bakulev and S.V. Mikhailov, Phys. Lett. B 436, 351 (1998).

[23] A.I. Vainshtein, V.I. Zakharov, V.A. Novikov, and M.A. Shifman, Sov. J. Nucl. Phys. 39, 77 (1984).

[24] F. Araki, M. Musakhanov, and H. Toki, hep-ph/9808290.

[25] I.I. Balitsky, Nucl. Phys. B254, 166 (1985), for a more general coordinate gauge; see S.V. Ivanov and G.P. Korchemsky, Phys. Lett. 154B, 197 (1985); S.V. Ivanov, G.P. Korchemsky, and A.V. Radyushkin, Sov. J. Nucl. Phys. 44, 145 (1986); V.I. Shevchenko and Yu.A. Simonov, Phys. Lett. B 437, 146 (1998).

[26] H.G. Dosch and Yu.A. Simonov, Phys. Lett. B 205, 339 (1988). 
[27] Yu.A. Simonov, Nucl. Phys. B307, 512 (1988).

[28] Yu.A. Simonov and J.A. Tjon, Ann. Phys. (N.Y.) 228, 1 (1993).

[29] A. Vainshtein and V. Zakharov, Phys. Lett. B 225, 415 (1989).

[30] D. Antonov, Surv. High Energy Phys. 14, 265 (2000).

[31] F.V. Gubarev, M.I. Polikarpov, and V.I. Zakharov, hep-th/9812030.

[32] R. Akhoury and V.I. Zakharov, Phys. Lett. B 438, 165 (1998); F.J. Yndurain, Nucl. Phys. B (Proc. Suppl.) 64, 433 (1998).

[33] G. 't Hooft, Nucl. Phys. B75, 461 (1974).

[34] E. Shuryak, Nucl. Phys. B198, 83 (1982); B203, 116 (1982).

[35] A.V. Radyushkin, Phys. Lett. B 271, 218 (1991).

[36] M.A. Shifman, hep-ph/9405246; hep-ph/9505289.

[37] I. Bigi, M.A. Shifman, N. Uraltsev, and A. Vainshtein, Phys. Rev. D 59, 054011 (1999).
[38] A.R. Zhitnitsky, Phys. Rev. D 53, 5821 (1996).

[39] A.M. Badalian and Yu.A. Simonov, Phys. At. Nucl. 60, 630 (1997).

[40] A.Yu. Dubin, A.B. Kaidalov, and Yu.A. Simonov, Phys. Lett. B 323, 41 (1994).

[41] D. La Course and M.G. Olsson, Phys. Rev. D 39, 2751 (1989); C. Olsson and M.G. Olsson, MAP/PH/76 1993.

[42] V.L. Morgunov, A.V. Nefediev, and Yu.A. Simonov, Phys. Lett. B 459, 653 (1999).

[43] S.I. Eidelman, L.M. Kurdadze, and A.I. Vainshtein, Phys. Lett. 82B, 278 (1979).

[44] Yu.A. Simonov, JETP Lett. 69, 505 (1999); Phys. Rep. 320, 265 (1999).

[45] H.G. Dosch, M. Eidemueller, M. Jamin, and E. Meggiolaro, J. High Energy Phys. 07, 023 (2000). 